\title{
What Do You See in the Cloud? Understanding the Cloud-Based User Experience through Practices
}

\author{
John C. Tang ${ }^{1}$, Jed R. Brubaker ${ }^{2,3}$, and Catherine C. Marshall ${ }^{3}$ \\ ${ }^{1}$ Microsoft Research, One Microsoft Way, Redmond, WA 98008 \\ ${ }^{2}$ Department of Informatics, University of California Irvine, Irvine, CA 92697 \\ ${ }^{3}$ Microsoft Research Silicon Valley, 1065 La Avenida, Mountain View, CA 94043 \\ \{johntang, cathymar\}@microsoft.com, jed.brubaker@uci.edu
}

\begin{abstract}
End users have begun to incorporate cloud-based services into their collaborative practices. What spurs and constrains this adoption? Are the cloud services understood adequately and used effectively? How might we intervene to promote a better connection between user practices and cloud services? In this study, we focus on collaborative practices that surround the adoption, use, and understanding of two popular, but sometimes contrasting, cloud services for creating and sharing content: Dropbox and Google Docs. We conducted 22 indepth interviews with people who used these services, including collaborators who used the services together, and people who had migrated from Google Docs to Google Drive. We found that users thought of the cloud in terms of the practices it helped them accomplish. Their understanding of the cloud was often shaped by the particular file storage and sharing technologies the cloud was replacing (remediation). Furthermore, collaborating with others through the cloud sometimes revealed different assumptions about how the cloud worked, leading users to develop socially negotiated practices around their use of the cloud. We use this analysis to identify some specific opportunities for designers to help users build more accurate conceptual models of the cloud and use its capabilities more fully: (1) when users are adopting the cloud to enact a practice; (2) when users are replacing an existing technology with the cloud; and (3) when users are encountering others' practices through collaboration.
\end{abstract}

Keywords: File synchronization, file sharing, online editors, collaboration, cloud user experience.

\section{$1 \quad$ Introduction}

Cloud-based file synchronizing (syncing) and sharing services have become a central element of everyday computing infrastructure. Ubiquitous access to storage, lowoverhead file sharing, coordination between devices, and real-time collaboration facilities have prompted significant end-user adoption of these services. These services, like other cloud computing technologies, are realizing Weiser's vision of ubiquitous computing, in which computation is accessible everywhere, but seamless and quiet in its presentation [21]. However, this invisibility may also present some challenges to users of cloud-based syncing and sharing services. Despite the availability of these 
services, research indicates that many people still harbor significant misconceptions about how these services work and may not understand the features they offer [16].

To identify opportunities for improving the design and uptake of cloud-based services, we investigated how users understand and incorporate the services in their individual and collaborative practices. In particular, we were interested in three areas: (1) how people adopt cloud-based syncing and sharing services; (2) how people work together through these cloud services, and how their understandings of the services influence those of their collaborators; and (3) how designers can pinpoint appropriate opportunities to help users better connect their practices with functionality offered by cloud services.

To give ourselves a concrete starting place, we focused on peoples' experiences with Dropbox and Google Docs. Both are widely used services that provide ubiquitous access to files and support collaboration through shared storage in the cloud, but they go about it in ways that put varying conceptual demands on their users. Google Docs offers a browser-based environment for access to and co-creation of content directly in the cloud [11]; users need only conceive of cloud repositories as a distinct shared place to do their work (although they must learn to use the new editors). By contrast, Dropbox synchronizes local files among devices and people to provide ubiquitous access to content [9]; while interaction with the editors and local file system is familiar, fully understanding Dropbox requires grappling with a complex notion of file synchronization. The recent release of Google Drive [1], which marries a Dropbox-like syncing client with the remote editing capabilities of Google Docs, provided us with a window onto how users' cloud experiences evolved as they migrated from Docs to Drive.

Our analysis draws support from Bolter and Grusin's theory of remediation [2]. Remediation highlights the relationship between media forms and their predecessors, and hints at how the new media forms are understood in terms of the old. Bolter explains, "designers of a new media form seek to borrow the cultural valence of one or more earlier forms. In most cases, however, they also want to define criteria by which the new form can surpass its predecessor, in order to give their audience a reason to adopt the new form." [3]

Remediation, which initially focused on media itself, has been extended to studies of media practice. According to Lanzara, "[t]he appearance of a new medium in a domain of practice produces a perturbation in the complex ecology of agents and activities, objects and tools, uses and meanings that constitute the practice." [15] In this study, we examine how individuals make sense of cloud services and incorporate them into their practices by applying their understandings of the services' remediated predecessors. As with other forms of infrastructure, these new services are "always built on an installed base" [4], so that individuals can scaffold their understanding of these technologies using experiences with their predecessors.

We begin with a brief description of the study and its participants. Next we organize our results according to two central themes: service adoption and reconciling collaborators' varying conceptual understandings the services. In the discussion section, we synthesize these stories into key findings, focusing on the relationship between cloud technologies and existing and developing social practices. We conclude with implications of our findings, highlighting key challenges in both the design of cloudbased services and opportunities to educate users how to better incorporate the cloud in their practices. 


\section{Study Description}

We conducted semi-structured, open-ended interviews with 22 people who use Drop-box and Google Docs (and in some cases, Google Drive) in individual and collaborative situations. Table 1 summarizes each participant's pseudonym, age, gender, background, and cross-references which collaborators we interviewed. Participants were primarily U.S. based, although one participant was from Canada and another was from New Zealand; they had used the services for between 3 months and 6 years.

Table 1. Summary of participant characteristics and pseudonyms. Participants were recruited through the authors' social media networks; direct contacts were excluded from participation.

\begin{tabular}{|c|c|c|c|c|}
\hline Pseudonym & Gender & Age & Occupation & Collaborators \\
\hline Dean & M & 33 & Artist and arts administrator & James \\
\hline Mary & $\mathrm{F}$ & 27 & Graduate student & \\
\hline Melissa & $\mathrm{F}$ & 37 & Graduate student & \\
\hline Nathan & M & 29 & Graduate student & \\
\hline Lance & M & 31 & Web developer & \\
\hline Aaron & M & 31 & Research assistant/Grad student & $\mathrm{Sao}, \mathrm{Xu}$ \\
\hline Trisha & $\mathrm{F}$ & 31 & Program manager at a non-profit & \\
\hline Jacob & M & 29 & Interaction designer, & \\
\hline Sao & $\mathrm{F}$ & 30 & Graduate student & Aaron, $\mathrm{Xu}$ \\
\hline Karen & $\mathrm{F}$ & 23 & Graduate student & Steve \\
\hline Sarah & $\mathrm{F}$ & 28 & Graduate student & Sanjay \\
\hline $\mathbf{X u}$ & M & 30 & Graduate student & Sao, Aaron \\
\hline Martha & $\mathrm{F}$ & 45 & Program manager & \\
\hline Dillan & M & 49 & Archivist & \\
\hline Sanjay & M & 25 & Graduate student & Sarah \\
\hline Otis & M & 37 & Systems analyst for an oil refinery & \\
\hline James & M & 36 & Online public relations & Dean \\
\hline Gary & M & 51 & Urgent-care physician & \\
\hline Andy & M & 36 & Field service technician & \\
\hline Steve & M & 25 & Software engineer /IT & Karen \\
\hline Jayden & M & 33 & Graduate Student & \\
\hline Bruce & M & 40 & Project lead/organizer & \\
\hline
\end{tabular}

Interviews lasted from 30 to 120 minutes (60 minutes on average) and were conducted over video chat or phone. The interviews focused on participants' adoption and ongoing experiences with Google Docs and Dropbox, as well as how their use of these services changed over time. Because some participants had been using either or both services for a long time, we asked them to find their oldest files in each service's store to help them recall early experiences. At the end of the interview, they were asked to summarize each product and compare them. 
Because we were interested in how collaborators influenced each other's understanding and use of the cloud services, we asked participants if we could contact the other people involved in the stories they told during their interviews. Five of the 22 participants were recruited this way. In one case, we interviewed three members of the same group; the others were dyads (who were sometimes part of larger groups).

Since our initial participants (17/22) did not have significant experiences with Google Drive's recently introduced local syncing client, we specifically recruited a final set of participants $(5 / 22)$ who had migrated to it. Given the substantial change in the conceptual model from Google Docs to Drive, we thought it was an interesting opportunity to see how users reacted to the availability of a local sync client. These interviews were conducted the same way as the others, except that Drive was included in participants' comparisons of cloud services.

All interviews were transcribed and participants were assigned the pseudonyms that are used in this paper. The interviewer (the second author) briefed the other authors about participant narratives immediately after the interviews took place. The analytical coding was performed jointly among the three authors; additional review of the transcripts was performed as necessary so that everyone was familiar with the details of the interview data. We performed inductive analyses of the interviews using grounded methods. Using open coding and memoing practices (as described in [5]), we grouped individual participant narratives into preliminary categories and labeled them. Labels and categories were in part emergent and in part influenced by our a priori interests in the adoption and collaborative use of these services, as well as the impact they had on existing practices. These categories were then refined using a constant comparison method that "combines inductive category coding with a simultaneous comparison of all social incidents observed" [10]. We also examined the interview data on a per-participant and per-collaboration basis to reveal adoption and use storylines and specific instances of remediation.

\section{Results}

In this section, we describe how participants develop their understanding of cloud syncing and sharing services and the ramifications of these understandings. We begin by exploring how participants adopt the services, either in response to problems that arose in the course of existing work practices, or through changes in the constellation of devices they use. We then show how the understandings and practices established during adoption affect ongoing use. Finally, we demonstrate how previous experiences with remote storage technologies produced some subtle misconceptions for participants, and examine the implications of these misconceptions for individual and collaborative use.

\subsection{Adoption as Problem Solving}

Participants described first learning about these services via online blogs (e.g., TechCrunch), from their peers, or in the context of project work with colleagues. Most 
were vaguely aware of these services prior to using them. For example, Dillan and Lance both reported that they were first introduced to Google Docs when Gmail opened attachments in it. For most participants, however, meaningful adoption (as opposed to brief engagement) was the result of "upgrading" their way of doing things to overcome specific limitations or cumbersome aspects of their current practice, which in turn influenced and constrained how they interpreted the technology.

For example, Dean adopted Dropbox to overcome email restrictions. As a graphic designer, he often needs to send remote collaborators Photoshop and Illustrator files that are too large for email attachments: "It was just an easy way to share files, rather than having to use YouSendIt, or WhaleMail, or any of those other sort of temporary big file e-mail systems."

For some, the limitations are latent. Sanjay, for example, was aware of Google Docs' centralized approach and facilities for real time collaboration. But it was only after he started co-authoring a report with a colleague in another country that he found the motivation to adopt the service. Limitations may prompt the adoption of more than one cloud service. Sanjay later adopted Dropbox too so he could automatically share reports saved to his local file system.

As Sanjay's scenarios demonstrate, adoption was often spurred by collaborative project work. The initial collaborations reported were often small, ad hoc, projectspecific, and rarely resulted in artifacts that were seen as archival. Even in more traditional professional environments, the collaborations remained lightweight (although adoption can be management-mandated). Trisha explained how her boss, who traveled, introduced her team to Dropbox, and how casual use became commonplace. Through use, Dropbox evolved from a literal dropbox to a shared repository.

We really use [Dropbox] a lot for... PowerPoint presentations that we can upload there so that when he's on the road ... he has access to our latest figures... And it started out from just like "Oh, we're just going to use it for a couple communication things or a couple staff documents," and now we're basically constantly sending everything into Dropbox. It's become our de facto shared drive.

Regardless of how individuals were introduced to the services, the motivation for their initial use shaped their subsequent understanding and expectations. Google Docs most frequently remediates editing suites like Microsoft Office, while Dropbox remediates storage and file transfer between devices or people, similar to uses identified by Dearman and Pierce [6]. As a result, subsequent use was often constrained to a narrow (albeit critical) set of cases.

\subsection{Adoption in Evolving Device Ecologies}

Not only did participants adopt cloud services to support existing practices when the old methods failed them; they also found themselves in situations in which evolving technological ecologies-including the introduction of new devices or the loss of old ones-prompted discovery and adoption of these services. 
The introduction of new mobile devices seems tied to cloud service adoption. This observation is well-aligned with the results of Sohn et al.'s study [20]. Participant Martha purchased a tablet to support her graduate studies; this in turn prompted her to adopt Dropbox when she found she was unable to use a thumb drive to transfer files to the device. She had learned about Dropbox from a blog post returned by a search for "How to get files onto iPad." Adding a device created a problem that was solved by adopting a cloud service.

New devices can also spur new practices, which in turn provide an opportunity for incorporating cloud services. This adoption path was common for our Google Drive participants. Bruce learned about Google Drive after finding it preinstalled on his new Android phone; to his delight, he discovered that he was able to pull up an agenda on his phone and conduct a meeting without a laptop. Similarly, Andy, a service technician, explained how important Google Drive had become for on-site access to notes, manuals, and other documentation:

At first it was just something that I installed just to see what it was all about, and then when I realized I could ... look at the same documentation on my laptop as I could on my phone... the phone has become this extremely useful tool.

Device loss was another change that prompted adoption. In Nathan's case, the loss of his USB drive forced him to reconceptualize the function of Dropbox. He had already adopted Dropbox for backup, but he still moved files between school and home using a thumb drive. This function only became apparent, however, when "I misplaced the USB at one point, and that's when I realized 'Oh, I'll just put it all on Dropbox." " Dropbox functionality did not change, but Nathan's practices (and thus his use) did.

New technology, as well as failures in existing technology, can promote adoption of cloud-based services and cause participants to reflect on what these services do. Hardware or new apps can present solutions to problems participants did not know that they had. Across our participants, we saw that understandings of these services most commonly broadened when participants' everyday practices were disrupted, and their focus was on the service itself, rather than the activity the service supports. By contrast, stand-alone information campaigns, such as those accompanying the introduction of Google Drive, did not seem to promote adoption or influence an individual's understanding of what Google's new service offered. Participants ascribed the migration to branding ("the logo changed") or found it inscrutable ("something about it is slightly different and I can't put my finger on it whatever that is"-Sarah)

\subsection{Conditional Adoption}

When participants described how they used the cloud, many saw the cloud through the lens of the limitations the services imposed and how they had changed the conditions of their work. This perception, in turn, either restricted the scope of their adoption (as when Google Docs replaced Word) or caused participants to compensate for disruptions in their normal practice (possibly by moving in and out of the service). 
Limited by the Basics. Because Google Docs remediated desktop editing suites (and not a shared document repository), many participants lamented the service's limited functionality and polish, and did not use it beyond simple documents like brainstorming output, meeting notes, and roommate expenses. For a few like Martha, who owned a small business, Google Docs was not viable for anything beyond notes: "[If] we're writing a proposal," she explained, "it has to look professional."

For those drawn into Google Docs for its synchronous collaboration functionality, a tension was created between it and the full-featured editor it was remediating. According to Karen, "The main issue is formatting... I have to copy and paste it all into Word. When you're getting towards the final revision, [Docs] just isn't feasible." Thus users adopted a cut-off point at which they would migrate from Docs back to a full-featured word processor to format the final document. However, this late-stage editing is often when the collaborative functionality of Google Docs is most needed. Some participants compensated for this by using Google Docs and shared Dropbox folders in tandem. Participants like Jacob described copying content from a Google Docs document into a Word file saved in Dropbox. Yet, while this approach retains some collaborative functionality, as both Martha and Melissa explained, using Dropbox this way limits concurrent access to the document.

Google Docs' inability to address the demands of pre-existing editing practices limited the extent to which participants were willing to commit to the service, and limited their willingness to acclimate to (and see the value of) Docs' novel functionality. Instead, participants were left weighing the benefits of Google Docs (often, the predicted contributions of collaborators) against its inability to format their content. The role of the remediated technology was clear: "Real time collaboration in Word would be the ideal world. I can't think of anything more fantastic than that." (Karen)

Acclimating to the Cloud. Participants discussed a variety of new practices they adopted to collaborate using cloud services. First, as other researchers have reported in their studies of shared repositories and workspaces $[8,18]$, the shared storage must be kept intelligible and consistent (so collaborators can find what they are looking for and negotiate practices to coordinate access so changes are not overwritten). Second, as we would expect from other studies of collaboration (e.g., [7]), collaborators must accommodate to a new level of visibility of their work and actions. This effect may become more pronounced when the collaborators are not peers (e.g., professors and their students, managers and their reports). The two effects are often intermingledthe document in its incoherent state is rendered abruptly available and visible by the new cloud services, either synchronously in Google Docs, or as an incomplete version when Dropbox syncs.

We observed multiple instances of how the remediated technology (in this example, Word) created an expectation of (and perhaps a genuine need for) change coordination facilities. Sarah described how her team's adoption of Google Docs resulted in breakdowns with one collaborator: "She always was worried that her work wasn't there or that she was being overwritten and she wasn't sure who was writing what."

Some participants coped with the perceived difference between their writing practices and those supported by the service by copying files in and out of the cloud. Sao, 
a graduate student, copied an important grant proposal out of the cloud and onto her local storage so her edits would not interfere with those of other collaborators. She explained that she prefers to do her initial drafts in Word, and then transfer her content into a shared Google Doc when she is ready to respond to feedback from her colleagues and make minor changes. Major edits, however, are always done outside of Google Docs:

Sometimes you will change a lot of things. And sometimes you want to take a long time to think about what you're going to write... So I first write on my own Word document and then later copy and paste to Google Docs.

Many participants commented on the authoring styles of their collaborators, which were abruptly rendered visible by the real-time collaboration in Docs, as well as the changes it caused in their own. Sanjay, while a strong advocate Google Docs' collaboration functionality, explained that under some circumstances he felt like he was under surveillance: "Let's say the deadline is tomorrow, the advisor is doing his part, and there is still things to do in my part. I can't sign off... it might look bad."

\subsection{Troubled Conceptions}

If participants' understandings of cloud syncing and sharing services develop in the context of the problems they solve or the practices and technologies they replace, and collaborative adoption proceeds in a hand-to-hand, viral way, then we might wonder about the effects of misconceptions: Do they hinder adoption? Do they limit successful use? Are they transmitted with the services? Do they lead to asymmetries in adoption, thus interfering with the overall benefit of services [12]? In this section, we explore the sources and effects of misconceptions.

“Cloud" Just Means Remote, Right? Thinking that files synced via Dropbox or Google Drive were not stored locally was a surprisingly common misunderstanding if participants had previous experience with remote or network storage. Dean, a graphic designer, harbored a misconception that stemmed from his prior use of an FTP server that he had set up to share files with his collaborators. Initially he described Dropbox as a portal between his computers, but later in the interview, he revealed that he uses Dropbox to "keep as much as possible off of my local hard drive" to prevent his primary computer, an aging laptop, from getting too bogged down, and that he assumed files that appeared to be local in the Dropbox folder actually lived in the cloud:

I assume that all of that shit lives on Dropbox's servers in the cloud and those [file icons] are basically links to the files... And if I just double click this text file I'm opening my text application and it's pulling the file from Dropbox's server. But the file is not living locally on my machine.

How do these misconceptions that an individual brings from experience with a remediated technology play into subsequent collaborations? Are these misconceptions corrected through interaction with collaborators, or do they remain in the background? We examine data from collaborations to find out. 
Conceptions in Collaboration. In individual use, incorrect or incomplete understandings of cloud services appeared to have limited implications. However, we had anticipated that these misconceptions would come to light during collaborations where users might encounter and be held accountable to the conceptual models maintained by their collaborators.

While we suspect that these conversations occur, the relative number of successes our participants reported in their collaborations struck us. One such surprise came when we interviewed Dean's primary collaborator, James. While James also had a somewhat incomplete understanding of Dropbox, his practices around the tool avoided any conflict with Dean. James copied files in and out of their shared Dropbox folder, and only edited them after copying them to a location outside of Dropbox. This practice enabled him to have a master and a renamed version with his changes:

If I'm editing a poster that Dean and I are working on together, if he puts [the file]... that he just touched, in [Dropbox], I'll copy it to my desktop and then open it in Photoshop. I want to preserve his changes, [but] I want to be able to delete stuff, or just have [a] backup, or make my changes. So I'll leave it [the original file], so it's like a primitive version control.

James subsequently complained that "Dropbox is... a little too much like a drawer, where something is either in or out of the drawer." Because James never edited a file in place in the shared Dropbox folder, he effectively avoided the very kind of version conflicts that might have caused Dean to reflect on his theory that he was working on a separate downloaded version of a remote file. Although these compatible misconceptions remained invisible in Dean and James' collaborations, the proliferation of files in their shared folder effectively exacerbated any problems Dean was experiencing as a result of a full hard drive.

By contrast, another collaboration (an academic research group that included participants Karen and Steve) negotiated a social workaround only after incompatible conceptions and misconceptions surfaced in a series of breakdowns. The group had reached a situation in which co-authors were working with out-of-date versions and overwriting one another's changes. These conflicts grew out of incompatible conceptual models. For Karen, Dropbox remediated a practice of emailing files to herself to ensure she had up-to-date versions on all of her computers:

\section{I have a computer in my lab at my university and I was using a laptop and then my personal desktop. ... Usually I was e-mailing things to myself. And if I for- got to e-mail it to myself or I e-mailed the wrong version then I was constantly redoing work I had already done because it was on the wrong machine.}

On the other hand, Steve (an undergrad who eventually administered the group's Dropbox folders) modeled his understanding of Dropbox on version control systems such as Subversion ${ }^{1}$. Subversion helped Steve make sense of how Dropbox syncs local and remote files, but also left him with the mistaken belief that Dropbox sometimes merged changed versions of files:

\footnotetext{
${ }^{1}$ http: //subversion. apache.org/
} 
The only thing... is [Dropbox] didn't really merge conflicts very well... if you and I are working on the same document at the same time and we both save it and it can't merge it together, it will save your copy as [AUTHOR NAME]'s conflicted copy and mine as Steve's conflicted copy.

Steve went on to provide a detailed (albeit mistaken) account of how Dropbox attempts to automatically merge changes and only produces conflict files when it is unsuccessful. This is similar to how code repositories like Subversion work: when changes are committed to the repository, any changes that can be merged automatically are. When they cannot be, users are asked to resolve conflicts before committing a particular file.

While Karen, who was also familiar with version control systems realized, "there's no real version control to merge things back together," Steve believed that the others in the group harbored misconceptions about Dropbox's ability to merge changes:

One of the professors...that [Karen] and I both worked under...didn't seem to understand that Dropbox was capable of merging. And so she would open up a document and resave it with her initials appended to it. And so over time you'd have eight documents with her initials on it and then somebody has to go through and merge her stuff into the original document when all she really had to do was open the original document. So she's a good example of not using it properly.

This was a complicated problem that involved both social components (the coauthors were not coordinating changes with one another) and technical ones (group members were syncing infrequently, in part because they had varying understandings of how to take better advantage of Dropbox). A breakdown over an important document (a grant proposal) led to the adoption of an explicitly social solution that did not resolve any (mis)conceptions, as Karen explains:

We have to call and say I'm working on this portion. This is how I'm going to save my folder or the document and what the name will be. ... So we actually include initials and time stamps and file names so that we have a way of tracking [who did what when].

Steve explained the resolution to the version conflict problem: in exchange for additional personal space on Dropbox, he became "the official Dropbox guy" who "had to merge crap all of the time. So once a week I'd go check it and say oh, you know, there's eight conflicts... and I would get rid of all of those." Just as in James and Dean's case, it was easier for participants to develop practice-based workarounds than it was to revise their mutual understanding of the cloud service. But in so doing, they missed an opportunity to learn how the cloud could better support their collaboration.

\section{Discussion}

What stands out in our results is the primacy of practices. They not only spur adoption-people adopt the cloud to solve problems-but they also suggest that people 
may use the cloud without really understanding how it works. In some ways, this is unsurprising: naturally people adopt practices, not features. And often they are using the cloud to remediate or maintain a practice, not a technology. But what we see is the consequential nature of what the cloud is replacing. If cloud syncing and sharing services are replacing FTP servers, then users are far more likely to understand the new service in terms of the old one. Their expectations are thus set, sometimes in ways that make them resistant to evolving understandings or extended use of these services. In this section, we reflect further on the relationship between user practices and cloud services, and examine more closely what this says about user education, addressing misconceptions, and the conditions for successful adoption and continuing use.

\subsection{Practices Overshadow the Cloud}

Across the board, participants reported that using Dropbox and Google Docs was straightforward and that the services were easily understood. However, participants often failed to see opportunities to expand their use of these services. This is in part because they focused on their work instead of on the tools they were using to do the work. Trisha provided an example of this tension when she explained that Dropbox would enable her team to maintain a single versioned master of a document "...instead of having 17,000 different versions of a single file as you update it, in theory there's one, although we actually have not been using it that way." Despite her awareness of this capability, her group continued to name versions: "it'll say like 'edits by' and then initials at the end, which is kind of silly... because then it's like you're using way more space than you need, but once people start doing something one way and get used to it, it's really hard to make them stop."

Google continues to add novel functionality to make its collaborative environment more robust. Yet, participants reported their dismay at the lack of support for the practices they already have - in the case of Google Docs, authoring content in a sufficiently robust client - more than their excitement for new modes of working. Our findings suggest that the inability of Google Docs to meet the standards of the technology it is remediating (i.e., the desktop office suite) limits most participants' desire to replace their existing tools with Google Docs, and for many, their ability to benefit from the collaborative features Docs provides.

Although these services have novel functionality that individuals' stories suggest they would find beneficial, they were largely unaware of this functionality. Instead, users focused on the essentials as defined by their tasks: Formatting and speed in Google Docs, and sharing files and managing storage quotas in Dropbox.

Understanding via Remediation. Bolter and Grusin assert, "Each new medium is justified because it fills a lack or repairs a fault in its predecessor, because it fulfills the unkept promise of an older medium." [2] Participants turned to remediated practices and technologies to make sense of the cloud. Projecting their understanding of how prior technologies enabled them to accomplish their practices onto how the cloud operates shapes the way they can understand the novel features that cloud-based 
services offer. The users' focus on their particular practice of interest narrows their view of what cloud services can do, which can in turn contribute to an incomplete or inaccurate conceptual model of how it works.

When to Educate Users. The ideal time to educate users about new functionality is when their focus is on practices associated with the tools and tasks in which they are engaged. For example, practices around using the tool, such as setup or maintaining the total storage within the tool's free quota, are when the user's attention is on the tool and not trying to accomplish a productivity task. Mary illustrates this point in describing how the Dropbox scavenger hunt helped her understand how to use it:

I told him to do the scavenger hunt, because not only do you get like the free storage, which was great, but then you also get to learn about like how to use it in like kind of a fun, interesting way where you're not just watching like a video, no matter how exciting they try and make it.

This Dropbox technique not only catches users when they are focused on managing the tool, but also gets users to enact practices they have learned (rather than just watching a video of them) to earn more storage space to ease their management work.

By contrast, Google's in-browser notifications about the transition from Docs to Drive, for example, were largely dismissed when they were encountered in the process of going to Google Docs to work on a file for a task. Furthermore, the wording of the transition notification ("Google Drive is the new home for Google Docs") suggests that no new practice is needed to migrate from Docs to Drive. Consequently, we found most users who had migrated to Drive were still using it in much the same way as they used Docs, without taking advantage of the new features that Drive offered.

Users were more likely to learn about the new features in Google Drive when they installed the mobile app (or found the app preinstalled) on their Android phone. At this point, they were focused on integrating a new mobile device or feature into their practices, making it an opportune time to learn more about how the cloud could help them. Taken together, these stories identify different types of teachable moments as users go about their work or as they manage cloud-based services.

\subsection{The Social Effects of Clouds}

Our previous study explored models [16]. For example, one collaborator would rely on a synced folder for archival storage, and the other would do housekeeping and delete a portion of this implicit archive. In this study we sought multiple perspectives on the same collaboration to breakdowns in participants' collaborations that could be attributed to the collaborators' conflicting conceptual see how they worked through these breakdowns.

While we continued to find examples of collaborators' conceptual mismatches, in this study, we were struck by how the participants worked around or managed their misconceptions. Generally, we found that many of the workarounds were social, and did not necessarily involve reconciling conflicting models. For example, Steve was 
designated as "the Dropbox guy" to resolve version conflicts and merge changes. It is probable that neither Steve, Karen, nor the professor revised their conceptual models.

We expected (or hoped) that encountering these kinds of problems as a group might provide teachable moments during which someone would invest the effort to understand how things are supposed to work and educate the rest of the group. Instead, we largely found that groups reverted to familiar practices that relied less on the cloud's ability to sync and manage changes and more on manually or socially managing updates, which they understood how to control. Thus, instead of refining or updating their collective conceptual model of cloud functionality, groups would construct social explanations and practices that avoid the features that they did not understand.

Compatible Misconceptions. In scenarios like Dean and James's, we see how two collaborators operate with conceptual misunderstandings that, nonetheless, do not result in breakdowns. While compatible misconceptions may be innocuous, during some interviews, it was clear that these misconceptions would likely lead to a breakdown in the future. For example, groups whose members believe that Dropbox supports simultaneous editing will eventually encounter conflicts where changes will need to be merged by hand.

Ad Hoc Collaborations. The majority of participants' cloud-based collaborations would best be described as "ad hoc." Since the cloud easily transcends organizational boundaries and firewalls, it is well-poised to facilitate ad hoc collaborations; in fact, the ability to cross firewalls is often a precondition for the adoption of syncing services [17]. As a result, two issues are worth noting: First, while Dropbox and Google Docs are often most productively used by groups, users may initially adopt these services individually.

Second, while these cloud services are often used outside traditional enterprise structures, they rely on collaborators having accounts with the services' providers. Participants often talked about the ease of using Dropbox and Google Docs for a quick project because "everyone had an account" (Aaron), but this technological ease can violate some implicit seams that participants use to productively distinguish one use venue from another. Trisha, for example, noted concerns about tools that spanned across work and home:

I didn't want to necessarily use it for my own personal stuff and accidentally save something in the work folder. Because when we first signed-up for Dropbox [at work], I was using it with my personal address because I already had an account, and I just didn't want to mix work and personal life more so than I'd already been doing.

These large-scale services appear to be enabling a broad set of new collaborative practices, but also present challenges for traditional enterprise work situations in which these services might not be viable. Perhaps to the dismay of network administrators, many participants in traditional enterprise settings spoke of using these tools to circumvent organizational policies to, as Lance said, "get things done." 


\section{Implications}

The adoption of Google Docs and Dropbox and the conceptual understandings individuals develop of the services highlight issues around when and how systems make features and processes visible, as well as how to connect these with users' practices. The drive towards seamlessness can leave users ill-equipped to adequately understand the nuanced behavior of their tools or make appropriate decisions when they encounter problems [19]. We make two arguments on this front: First, designers of cloudbased services can improve user experiences by exposing technical functionality and processes in relation to people's practices. Second, service providers can facilitate the development of cloud-based practices by taking advantage of teachable moments to educate users about their functionality.

\subsection{Seeing the Cloud through Practices}

Cloud services need to aid users in connecting their practices with what these cloud services are actually doing. As previous research has noted [16], the lack of process transparency may prevent individuals from broadly adopting cloud-based services. Our findings suggest that simply exposing the functional behavior of these services may be insufficient. Instead, designers should provide more robust user feedback on processes but in relationship to user practices and their working context and not in terms of system processes or features.

The Google Docs text editor provides an excellent example of how system processes can be exposed in a way that aligns with user practices. The addition of coauthors' cursors in Doc's word processor, for example, and synchronous appearance of a coauthor's text provide little room for ambiguity about the collaborative editor's functionality: co-authors are changing the same document. When and how Google Docs saves content is less clear. When connected to the Internet, saving is unimportant: Google automatically saves content as it is added. In fact, Google has removed "Save" buttons and menu options from their editors. Saving becomes ambiguous, however, when individuals proactively want to save their content - for example, when the user's Internet connection has been lost. In this case, Docs notifies the user that it is trying to reconnect to Google's servers, but does not explain how changes made in the meantime will be merged, or what options individuals have to preserve their work if the interruption in network connectivity persists.

Turning to Dropbox, we see a good example of how the process of creating a shared folder connects with user practices. When users share a folder (either directly on Dropbox's website or via their operating system's context menu), the user is presented with a webpage on which they can provide the email addresses of those they would like to invite to the folder. An invitee, upon receiving the email invitation, can click a link to accept the invitation, at which point the invitee is notified about the addition of the new shared folder both in a web browser and the local syncing client. When sharing a folder, the user's practice (e.g., email invitation, files appearing in a folder) and the system's functionality are highly aligned. 
However, once a folder is shared, functionality is far less clear. A number of participants described confusion about the relationship between their Dropbox folder and the rest of their hard drive. Dragging a file from Dropbox to another folder moves the file rather than copying it. James' comment about Dropbox acting "like a drawer" stems from this interaction. What is not immediately clear to users is that moving a file outside of Dropbox affects all collaborators - it effectively removes the file from everybody's Dropbox. Moreover, this breakdown was compounded when participants found social explanations for technical breakdowns. Dropbox could address this confusion by simply alerting users that moving files out of shared folders will remove access to those files by other collaborators.

Our implication that processes should be exposed relative to user practices presents some significant challenges for services that operate at either the system or the interface level. Dropbox, for example, intentionally limits the focus of its syncing client to the file system and remains agnostic to programs and user practices outside of how they read and write to the hard drive. Yet, many of the collaborative practices around using Dropbox involve editing those files using productivity tools. Since Dropbox's implementation does not afford providing any process transparency in the context of those editors, users can get confused about how Dropbox manages concurrent or conflicting changes while editing those files. Conversely, the web-based editors in Google Docs made it transparently obvious when concurrent editing occurred. Yet Google Docs removed commands for file management (no Save command), making it unclear how to save work when an Internet connection is interrupted or lost. Cloud service designers need to consider the full scope of user practices to help users understand how to fully integrate the cloud's capabilities into their work.

\subsection{Learning How to Look at Clouds}

Given the scope of changes to cloud syncing and sharing services over the last year, we expected to see user practices and understandings change in response. Instead, we found that participants' conceptualizations of these systems remained relatively unchanged. Since users' practices have been established and largely remained stable, changes in the cloud services by themselves were not enough to engage users in the work of learning about them.

Revisiting an earlier example, it was noticeable how most of our participants - active users of cloud services - were unaware of the particulars around the transition from Google Docs to Google Drive, despite prominent notifications. Indeed, given the lack of immediate impact on their practices, many thought that the change to Drive was simply a rebranding of the service.

If our aim is to enable broader use of the cloud, our findings demonstrate that improving the design of cloud services alone is insufficient. Service providers must also educate users about how their practices can take greater advantage of the cloud. People may not notice new features even when they are prominently announced. Moreover, the rate at which the cloud services are changing challenges users' ability to keep up with those changes or even identify "expert users" to help work through conceptual breakdowns, as they have in the past [14]. Our study identified four different kinds of 
teachable moments when users were more amenable to learning about new features or capabilities of the cloud. We go through each type in turn.

Teachable Moments Around Adoption. Adoption presents a clear period during which initial understandings are being formed. Critical windows for forming understandings are not limited to service adoption. The addition of new devices also caused participants to reassess their use of cloud-based tools. Indeed, the most successful instances of Google Drive adoption reported by our participants were the result of discovering the Drive application pre-installed on a new mobile device.

Teachable Moments around Remediation. Services should expect and account for the fact that new users bring existing practices with them. By understanding which prior technologies and practices a cloud service remediates, providers can anticipate user expectations that can inhibit deep adoption; in this way, they can also prevent misconceptions. Perhaps providing different on-ramps tailored for the common tools that are being remediated by the cloud would help connect users' practices with cloud features of most interest to them, and would help anticipate and circumvent many standard misconceptions.

Teachable Moments around Cloud Management. User understandings were pliable when practices were focused on managing the services themselves. Dropbox's disk quota, for example, presents an overarching user problem that requires that heavy users "maintain" their storage from time to time. Dropbox rewards users with additional storage for completing a variety of tasks, most of which introduce users to Dropbox functionality or prompt them to tell others about Dropbox. Numerous participants described learning about Dropbox from its "Tour", "Simple tasks", and "Scavenger hunts." Dropbox takes advantage of a limit that they have imposed on their users to encourage them to learn more about the service and teach others about it.

Teachable Moments around Collaboration. Collaborating with others naturally exposes other people to the consequences of actions in the cloud. Updating or deleting files in the cloud will affect what others see. These collaboration consequences could be used to help users understand how the cloud is mediating their collaboration. Besides the visibility of concurrent editing in Google Docs, there are other ways in which the cloud could create teachable moments during collaboration. File deletion presents an opportunity to show users the effect of this action on their collaborators (i.e. they will no longer have access to it). Alerting users as to when and why conflict resolution files are created would help users understand the limits of synchronization. A "sync inspector" that more transparently indicates who last edited the file (and on which device) would help users have a better sense of how changes from various people and devices are being coordinated through the cloud (Cimetric [17] is an early attempt at providing such a sync inspector). Browsing sync history could also help users diagnose why they do not have access to the expected file version (for example, the one they saved on another device), since they might be able to identify when a sync was missed, which might help them narrow down possible causes for a sync 
failure. While collaboration naturally exercises many of the cloud features needed to keep files in sync, users need more help to observe and understand these processes to appreciate how the cloud is working to support collaboration.

\section{Conclusion}

Our study demonstrates the ways in which individuals adopt cloud-based syncing and collaboration services into their work practices. We found that practices from remediated technology fundamentally shaped the ways participants understood and used cloud functionality. Individuals adopt cloud services to solve problems or when incorporating new technology into their routines. However, when cloud services do not fully support users' existing practices, they may only partially adopt cloud functionality. Moreover, given the limited transparency of cloud processes, experience from remediated technologies or collaborating with others who have mismatched conceptions often resulted in misconceptions that also limited their use of the cloud.

We identify opportunities to improve cloud experiences, both by redesigning aspects of the services and by appropriately educating users about how their practices might benefit from the cloud. Designers of cloud-based services need to strike a balance between visibility and seamlessness. Decisions around visibility should be made based on users' practices and not the system processes. Perhaps the more salient opportunity stems from identifying specific teachable moments for educating users how to more fully incorporate the cloud into their practices. We identified these teachable moments as they occur during adoption, remediation, management, and collaboration. As cloud services and users' cloud practices continue to develop, we expect that these implications will help to improve current and future cloud services.

\section{References}

1. Biggs, J.: Google Drive Is Live With 5GB Of Free Storage And Google Docs Hoo-kup. TechCrunch (2012), http: //techcrunch.com/2012/04/24/google-drive-is-live/

2. Bolter, J., Grusin, R.: Remediation: Understanding New Media. MIT Press, Cambridge (1999)

3. Bolter, J.D.: Formal Analysis and Cultural Critique in Digital Media Theory. Convergence: The International Journal of Research into New Media Technologies 8(4), 77-88 (2002)

4. Bowker, G.C., Star, S.L.: Sorting Things Out. MIT Press, Cambridge (1999)

5. Corbin, J., Strauss, A.L.: Basics of qualitative research. Sage, Newbury Park (2008)

6. Dearman, D., Pierce, J.S.: "It's on my other computer!": Computing with Multiple Devices. In: Proc. CHI 2008, pp. 1144-1153 (2008)

7. Dourish, P., Bellotti, V.: Awareness and coordination in shared workspaces. In: Proc. CSCW 1992, pp. 107-114 (1992)

8. Dourish, P., Lamping, J., Rodden, T.: Building bridges: Customization and mutual intelligibility in shared category management. Group 1999, 11-20 (1999)

9. Dropbox. What is Dropbox (2012), https://www. dropbox.com/tour/1 
10. Goetz, J.P., LeCompte, M.D.: Ethnographic Research and the Problem of Data Reduction. Anthropology \& Education Quarterly 12(1), 51-70 (1981)

11. Google. An overview of Google Docs (2012), http: / / support.google.com/docs/bin/ answer . py $? h l=e n \& a n$ swer $=49008$

12. Grudin, J.: Groupware and social dynamics: Eight challenges for developers. CACM 37(1), 92-105 (1994)

13. Hamlen, K.W., Liu, P., Kantarcioglu, M., Thuaisingham, B., Yu, T.: Identity management for cloud computing: Developments and directions. In: Workshop on Cyber Security and Information Intelligence Research (2011)

14. Kellogg, W.A., Breen, T.J.: Evaluating user and system models: Applying scaling techniques to problems in human-computer interaction. In: CHI 1987, pp. 303-308. ACM Press (1987)

15. Lanzara, G.F.: Remediation of practices: How new media change the ways we see and do things in practical domains. First Monday 15(6-7) (June 2010)

16. Marshall, C., Tang, J.C.: That syncing feeling. In: Proceedings of Designing Inter-active Systems Conference (DIS 2012), pp. 544-553. ACM Press (2012)

17. Marshall, C., Wobber, T., Ramasubramanian, R., Terry, D.: Supporting Research Collaboration Through Bi-Level File Synchronization. In: Proc. Group 2012, pp. 165-174. ACM Press (2012)

18. Rader, Emilee, Yours, Mine, and (Not) Ours: Social Influences on Group Information Repositories. In: Proc. CHI 2009, pp. 2095-2098 (2009)

19. Ratto, M.: The Ethics of Seamlessness: Resources and Future Directions. International Review of Information Ethics 8(12), 20-27 (2007)

20. Sohn, T., Li, K.A., Griswold, W.G., Hollan, J.D.: A Diary Study of Mobile Information Needs. In: Proc. CHI 2008, pp. 433-442 (2008)

21. Weiser, M.: The computer for the 21st century. Scientific American 265(30), 94-104 (1991) 